\title{
Reabilitação oral com implante e carga imediata unitária por cirurgia guiada: relato
}

\section{de caso}

\author{
Oral rehabilitation with dental implant and immediate loading by guided surgery: case report \\ Rehabilitación oral con implante dental y carga inmediata mediante cirugía guiada: reporte de caso
}

Recebido: 02/12/2020 | Revisado: 10/12/2020 | Aceito: 29/12/2020 | Publicado: 02/01/2021

\author{
Aretha Heitor Veríssimo \\ ORCID: https://orcid.org/0000-0002-7951-8843 \\ Universidade Potiguar, Brasil \\ E-mail: aretha.heitor@gmail.com \\ Juliana Augusta Nascimento de Souza \\ ORCID: https://orcid.org/0000-0002-3025-8091 \\ Universidade Potiguar, Brasil \\ E-mail: julianasouzaodontologia@gmail.com \\ Taynnah Alves de Oliveira \\ ORCID: https://orcid.org/0000-0001-8721-3604 \\ Universidade Potiguar, Brasil \\ E-mail: taynnah.a@hotmail.com \\ Alana Ghiotto Gonçalves \\ ORCID: https://orcid.org/0000-0002-6208-6597 \\ Universidade Potiguar, Brasil \\ E-mail: alanaghiotto@outlook.com \\ Felipe Alexander Caldas Afonso \\ ORCID: https://orcid.org/0000-0001-9808-8714 \\ Universidade Potiguar, Brasil \\ E-mail: felipealexander@gmail.com \\ Francisco de Assis de Souza Junior \\ ORCID: https://orcid.org/0000-0003-3834-2690 \\ Universidade Potiguar, Brasil \\ E-mail: souzajuniorfa@ hotmail.com
}

\begin{abstract}
Resumo
O edentulismo é um desafio extremamente frequente no dia-a-dia do cirurgião dentista. A busca de soluções funcionais e estéticas para estes casos têm proporcionado diversas inovações no mercado de materiais odontológicos como, por exemplo, os implantes dentais osseointegráveis, próteses, coroas e guias cirúrgicas. O aprimoramento de materiais e métodos em Implantodontia trouxeram possibilidades de intervenção, que por meio do planejamento digital e confecção de guias cirúrgicas 3D, concedem menor tempo de trabalho para assim, ofertar ao paciente maior conforto através de técnicas menos invasivas e cruentas, com a finalidade de reestabelecer a função, estética e condição biopsicossocial do paciente. Este caso clínico visa descrever o planejamento e instalação de implante dentário cone morse imediato (Drive, Neodent ${ }^{\circledR}$, Curitiba, Brasil) utilizando a técnica com guia cirúrgica 3D (3D Studio - Planejamento Virtual da Face ${ }^{\circ}$, Natal- RN, Brasil), planejada através de tomografia computadorizada cone beam analisada através do software Blue Sky Bio LLC (Libertyville, Illinois), além da realização de enxertia com estrutura óssea mineral bovina (Lumina-Bone Fino, Criteria®, SP- São Paulo, Brasil) seguido da confecção de coroa unitária e instalação de carga imediata em área estética.

Palavras-chave: Implantes dentários; Osseointegração; Prótese dentária; Cirurgia assistida por computador; Estética.
\end{abstract}

\begin{abstract}
Edentulism is an extremely frequent challenge in the dentist's daily routine. The search for functional and aesthetic solutions for these cases has led to several innovations in the dental materials market, such as osseointegrated dental implants, prostheses, crowns and surgical guides. The improvement of materials and methods in Implantology brought possibilities for intervention, which through digital planning and preparation of 3D surgical guides, grant less work time to offer the patient greater comfort through less invasive and bloody techniques, with the purpose to reestablish the patient's function, aesthetics and biopsychosocial condition. This clinical case aims to describe the planning and

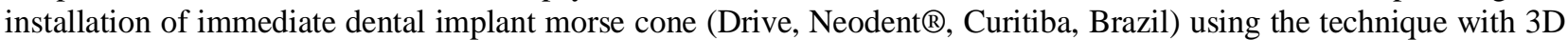
surgical guide (3D Studio - Virtual Face Planning®, Natal-RN, Brazil), planned through cone beam computed tomography analyzed using Blue Sky Bio LLC software (Libertyville, Illinois), in addition to grafting with bovine mineral bone structure (Lumina-Bone Fino, Criteria ${ }^{\circledR}$, SP- São Paulo, Brazil) followed by unitary crown and installation of immediate load in aesthetic area.
\end{abstract}

Keywords: Dental implants; Osseointegration; Dental prothesis; Surgery computer-assisted; Esthetics. 


\begin{abstract}
Resumen
El edentulismo es un desafío extremadamente frecuente en la rutina diaria del dentista. La búsqueda de soluciones funcionales y estéticas para estos casos ha dado lugar a diversas innovaciones en el mercado de materiales dentales, como implantes dentales osteointegrados, prótesis, coronas y guías quirúrgicas. La mejora de materiales y métodos en Implantología trajo posibilidades de intervención, que a través de la planificación digital y elaboración de guías quirúrgicas 3D, otorgan menor tiempo de trabajo para ofrecer al paciente mayor comodidad mediante técnicas menos invasivas y sanguinolentas, con el propósito restablecer la función, estética y condición biopsicosocial del paciente. Este caso clínico tiene como objetivo describir la planificación e instalación de cono morse de implante dental inmediato (Drive, Neodent ${ }^{\circledR}$, Curitiba, Brasil) utilizando la técnica con guía quirúrgica 3D (3D Studio - Virtual Face Planning®, Natal-RN, Brasil), planificado mediante tomografía computarizada de haz cónico analizada con el software Blue Sky Bio LLC (Libertyville, Illinois), además del injerto con estructura ósea mineral bovina (LuminaBone Fino, Criteria ${ }^{\circledR}$, SP- São Paulo, Brasil) seguido de Corona unitaria e instalación de carga inmediata en zona estética.
\end{abstract}

Palabras clave: Implantes dentales; Osteointegración; Prótesis dental; Cirurgía asistida por computador; Estética.

\title{
1. Introdução
}

O desenvolvimento dos implantes osseointegráveis por Brànemark em 1969 concedeu um grande avanço na cirurgia odontológica, pois proporcionou elevado nível de previsibilidade e sucesso clínico através do protocolo de dois estágios cirúrgicos, separados por um período de reparo responsável pela estabilização da interface osso/implante antes do carregamento protético funcional para casos de desdentado total ou perdas dentárias parciais. (Kayatt et al., 2008; Branemark et al., 1977)

A instalação de implantes osseointegrados como terapêutica reabilitadora oral é considerada como um tratamento seguro e previsível tanto para pacientes desdentados parciais como para totais (Muddugangadhar, 2015). Em que, os implantes trouxeram novas possibilidades para as reabilitações dentárias, sendo a tecnologia dos programas computacionais, associada à aquisição de imagens médicas (tomografia computacional), que geram imagens tridimensionais de alta qualidade podem permitir a visualização, análise e manipulação de estruturas anatômicas auxiliando o planeamento reabilitador. (Guerra, 2017; Cauduro et al., 2019). Dessa forma, vários programas e softwares vêm sendo utilizados na implantodontia para planejamento cirúrgico de implantes dentais. Sendo, as três maneiras mais comuns de aplicar este planejamento cirúrgico em um ambiente clínico da cirurgia guiada, as guias de perfuração processadas por prototipagem rápida, guias fabricados por fresadoras e por meio de navegação cirúrgica. (D’haese et al., 2016)

Em instalações de implante imediato com carga imediata, o planejamento prévio possibilita a confecção do guia multifuncional e prótese provisória fabricadas nas formas e posições mais adequadas para estética e função. Tendo em vista, que na ausência desse planejamento, o posicionamento do implante e coroa será realizado de forma empírica, porque não haveria a realização de enceramentos ou designer prévio do posicionamento ou formato para orientação do procedimento reabilitador (Borges et al., 2014). Sua aplicação busca menor número de intervenções cirúrgicas e redução do tempo de tratamento, assim promovendo maior satisfação do paciente. Os critérios que se deve levar em conta para a indicação e utilização de implantes com carga imediata são a qualidade do tecido ósseo, propriedades macroscópicas e microscópicas dos implantes, estabilização bicortical, distribuição dos implantes, além do uso cauteloso de cantilevers. (Kayatt et al., 2008; Schnitman, Wohrle \& Rubenstein, 1990)

Os enxertos exógenos encontram-se disponíveis em blocos e também em grânulos cortical ou esponjoso. Possuem indicação para o tratamento de aumento ou reconstrução do rebordo alveolar, preenchimento de defeitos intra-ósseos e de alvéolos dentários, visando a preservação do rebordo alveolar, elevação do seio maxilar, preenchimento de defeitos periodontais associados a regeneração óssea guiada e preenchimento de defeitos periimplantares. Em relação as suas propriedades, tem a capacidade de promover uma melhor revascularização e, ainda, mantém um arcabouço para a osteocondutividade, aumentando a estabilização do coágulo e absorção sanguínea natural entre os micros e macroporos. Com 
isso, configura-se como um excelente material para uso na implantodontia oral. (Ferreira, Dalapicula, Conz \& Vidigal Junior, 2007)

Em relação às condições de carga, é necessário um período de 5 a 6 meses para a osseointegração dos implantes para maxila e 3 a 4 meses para mandíbula. No entanto, a osseointegração com carregamento precoce também é uma possibilidade, como demonstrado em estudos recentes. (Brenemark et al., 1977) O tipo de conexão e as condições de carga afetam diretamente o sucesso da reabilitação. As conexões do tipo Cone Morse apresentam vantagens sobre as demais conexões, por promover maior vedação e proporcionar maior estabilidade. Isso ocorre por essa conexão fornecer contato próximo na interface implante/pilar, proporcionando maior estabilidade. (Oliveira et al., 2017; Takahashi et al., 2015; Rismanchian, Bojohli, Ghotamreza \& Razavi, 2014; Sanz-Sánchez, Sanz-Martín, Figuero \& Sanz, 2015)

Sendo assim, a técnica do implante imediato juntamente com a coroa provisória imediata tornou-se uma opção bastante segura para a reabilitação oral. Este relato de caso descreve o planejamento e instalação de implante dentário imediato do tipo cone morse (Alvin, Neodent ${ }^{\circledR}$, Curitiba, Brasil) utilizando a técnica guiada com guia cirúrgica 3D (3D Studio Planejamento Virtual da Face ${ }^{\circledR}$, Natal - RN, Brasil), planejada através de tomografia computadorizada cone bean, que foi analisada através do software Blue Sky Bio LLC (Libertyville, Illinois), além da realização de enxertia com osso particulado mineral bovino (Lumina-Bone Fino 0,5g - Criteria®, SP- São Paulo, Brasil) seguido da confecção e instalação de coroa provisória com carga imediata em área estética.

\section{Metodologia}

O presente estudo se propõe a apresentar uma descrição da técnica do implante imediato juntamente com a coroa provisória imediata por meio de relato de caso clínico (estudo de caso) com objetivos descritivos, exploratórios, com abordagem qualitativa. (Pereira et al., 2018). Assim, de acordo com o conhecimento da prática clínica e com base na literatura científica sobre o tema abordado, e considerando as diferentes possibilidades de condução do caso apresentado, propôs-se realizar uma reabilitação oral com implante e carga imediata unitária por cirurgia guiada. O paciente em questão assinou o Termo de Consentimento, permitindo a participação no estudo anteriormente ao início do tratamento, assim como os procedimentos seguidos estavam de acordo com os padrões éticos em pesquisa humana segundo de acordo com a Declaração de Helsinque de 1975, conforme revisada em 1983.

\section{Relato de Caso}

Paciente masculino J.S.M., 43 anos, procurou atendimento odontológico na Clínica Solon Galvão (Universidade Potiguar - UnP, Natal-RN, Brasil) com queixa principal nos elementos 14, 15 e 25. Relatou que necessitava de implante nestes elementos, sendo o elemento 14 um remanescente radicular. Realizada anamnese, exame físico e tomadas fotográficas extra e intra-oral. Para complementar a documentação necessária ao planejamento cirúrgico virtual, o paciente foi submetido à tomografia computadorizada cone bean de maxila (Figura 1). No planejamento do elemento 14, foi observado que havia manutenção da crista óssea, volume ósseo vestibular, avaliação da linha do sorriso, condição periodontal, papilas e mucosas adjacentes.

Após o planejamento e avaliação da necessidade de estética imediata do paciente, foi confirmada a possibilidade do uso da técnica de exodontia do remanescente radicular, juntamente com instalação do implante imediato através da cirurgia guiada e confecção de provisório em caso de torque igual ou maior que 32N. 
Figura 1. Imagem tomográfica do elemento 14, a qual podemos observar a raiz do elemento em questão, assim como a manutenção da crista óssea e volume ósseo vestibular.

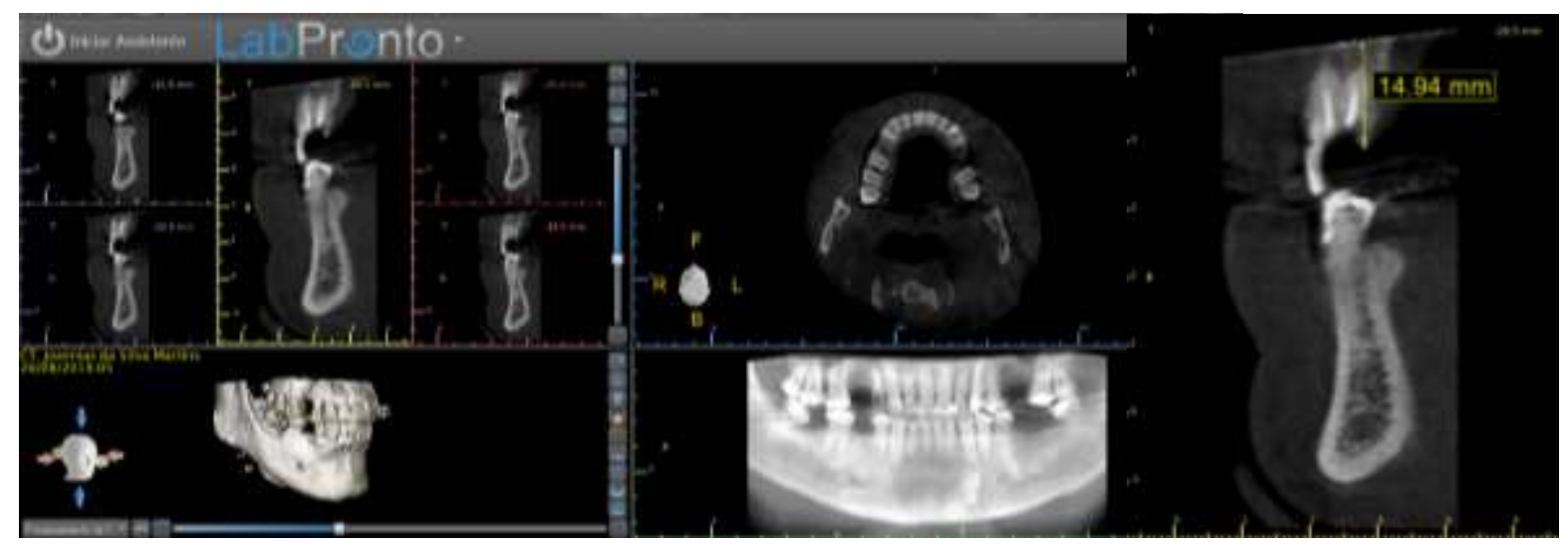

Fonte: Autores (2020).

\subsection{Confecção do Guia Cirúrgico}

Foi realizada moldagem da arcada superior do paciente para confecção de modelo em gesso, o qual foi escaneado para gerar imagens em STL auxiliar no planejamento virtual cirúrgico. Juntamente com a tomografia computadorizada cone bean de maxila, pôde-se observar disponibilidade óssea para instalação de um implante Cone Morse Drive de 3,5 mm x $10 \mathrm{~mm}$ (Neodent ${ }^{\circledR}$, Curitiba-PR, Brasil). Dessa forma, foi panejado e confeccionado um guia cirúrgico por prototipagem em impressora 3D (3D STUDIO - Planejamento Virtual da Face ${ }^{\circledR}$, Natal-RN, Brasil) (Figura 2).

Figura 2. A: Guia cirúrgico sendo planejado virtualmente; B: Guia cirúrgico impresso por prototipagem em impressora 3D (3D STUDIO - Planejamento Virtual da Face®) apresentando as anilhas para perfuração.

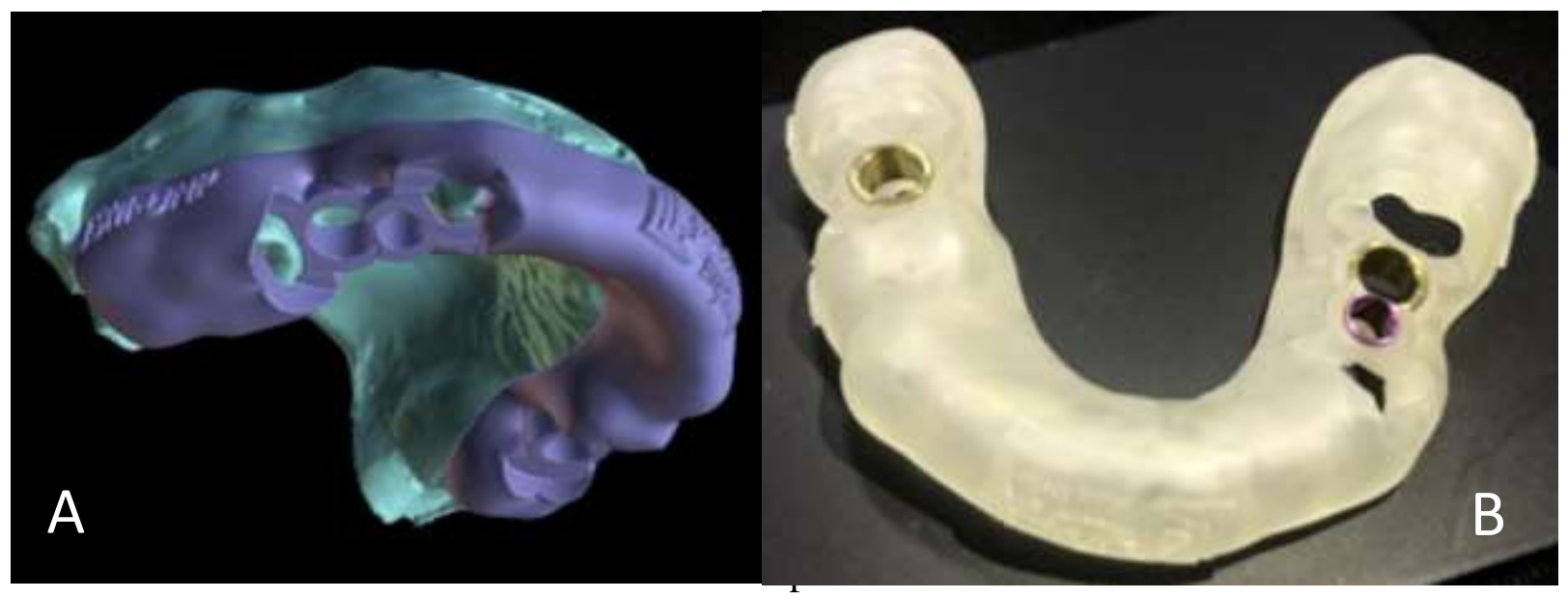

Fonte: Autores (2020).

\subsection{Fase Cirúrgica}

Inicialmente, realizou-se assepsia intra e extra oral, anestesia infiltrativa em fundo de vestíbulo e por palatina em área do elemento 14 , seguido por sindesmotomia e exérese com extrator reto, na finalidade de não romper o contorno gengival e manter a papila em posição (Figura 3), evitando perda óssea, e realizando a exodontia atraumática sem retalho do elemento. 
Figura 3. Elemento 14 antes (A) e após (B) exodontia sem retalho, sem rompimento do contorno gengival e mantendo a papila em posição, observar a manutenção dos tecidos.

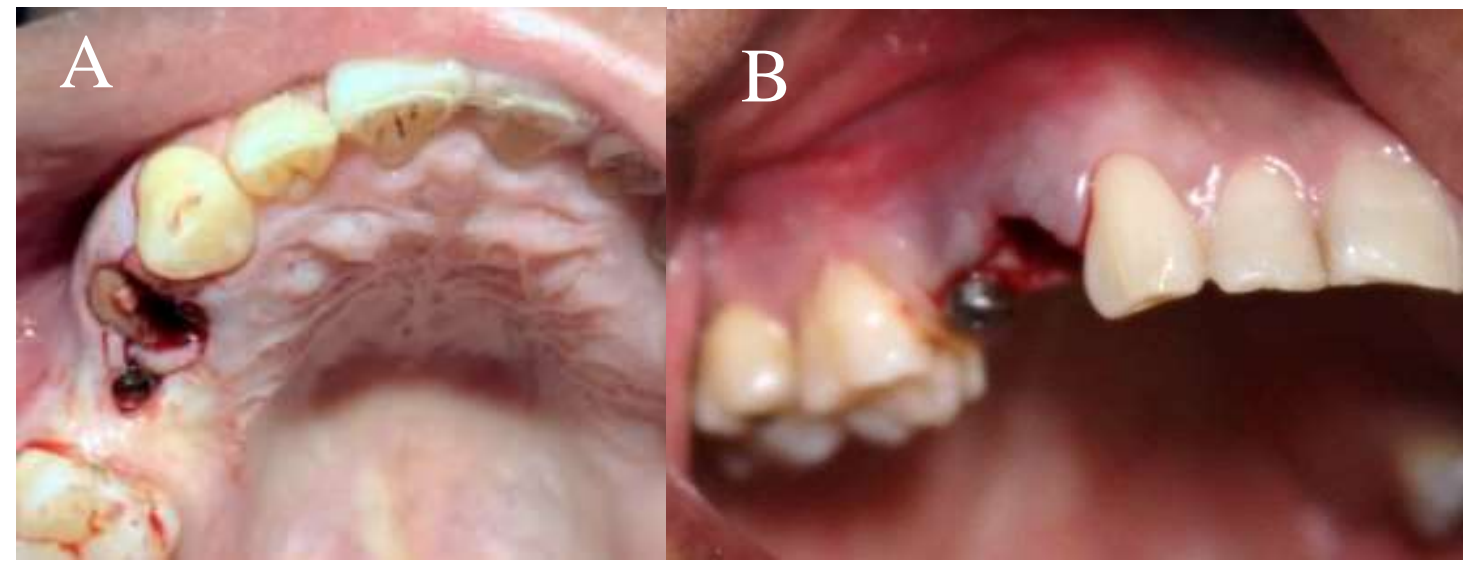

Fonte: Autores (2020).

Após a exodontia, foi utilizado o guia cirúrgico (3D STUDIO - Planejamento Virtual da Face®, Natal-RN, Brasil), anteriormente planejado, para auxiliar na osteotomia do osso alveolar, sempre avaliando a posição ideal do implante associada à reabilitação futura (Figura 4). As anilhas Cone Morse Guided Surgery Neodent®, Curitiba-PR, Brasil) (Figura 5) utilizadas no guia cirúrgico foi de modelo estreita (roxo), com diâmetro interno de 3,5 mm (Neodent $®$, Curitiba-PR, Brasil).

Figura 4. Guia cirúrgico (3D STUDIO - Planejamento Virtual da Face ${ }^{\circledR}$, Natal-RN, Brasil) posicionado intra-oral para perfuração do alvéolo observar os posicionamento atrás das anilhas.



Fonte: Autores (2020). 
Figura 5. Kit cirúrgico Cone Morse Guided Surgery (Neodent ${ }^{\text {, }}$, Curitiba-PR, Brasil) contendo as anilhas e brocas de perfuração utilizadas juntamente com o guia cirúrgico para guiar as perfurações e instalação do implante dentário.

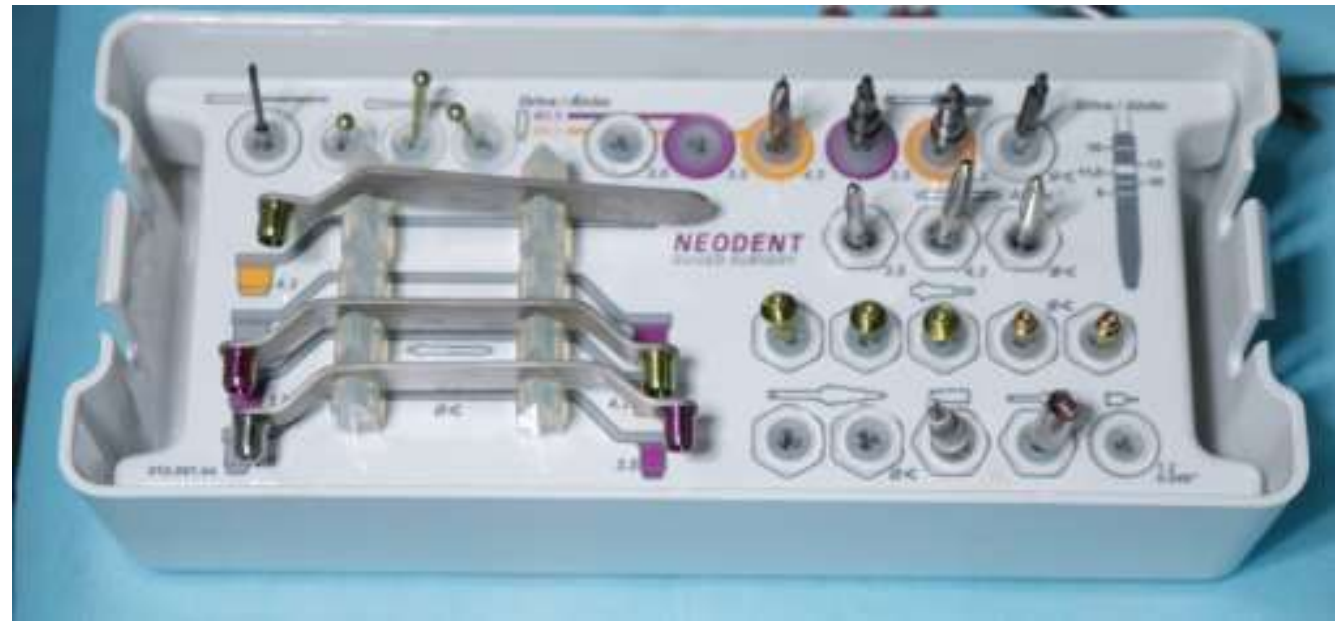

Fonte: Autores (2020).

As perfurações seguiram o protocolo de diâmetros progressivos, atentando-se para o posicionamento mesiodistal e vestibulolingual do implante, distando dos elementos dentários proximais no mínimo 3mm. A perfuração foi iniciada com a broca lança na parede palatina com perfuração de $12 \mathrm{~mm}$, preservando a parede vestibular. Em seguida, foram utilizadas as brocas de perfuração sequenciais, sendo as brocas broca 2,0 $\mathrm{mm}$ perfurando $12 \mathrm{~mm}$ e por último broca $3,5 \mathrm{~mm}$ perfurando 10mm. Esta conduta permitiu a instalação do implante Cone Morse Drive de 3,5 mm x 10 mm (Neodent®, Curitiba-PR, Brasil) (Figura 6) com um torque de $45 \mathrm{~N}$, com a realização do provisório imediato sobre munhão universal (3,3x6mm, Neodent®, Curitiba-PR, Brasil). O alvéolo foi preenchido com osso particulado mineral bovino (Lumina-Bone Fino 0,5g - Criteriaß, São Paulo-SP, Brasil) (Figura 7).

Figura 6. Instalação do implante Cone Morse Drive de 3,5 mm x 10 mm (Neodent ${ }^{\circ}$, Curitiba-PR, Brasil) com auxílio do Guia cirúrgico (3D STUDIO - Planejamento Virtual da Face ${ }^{\circledR}$, Natal-RN, Brasil), observar posicionamento guiado pela anilha.

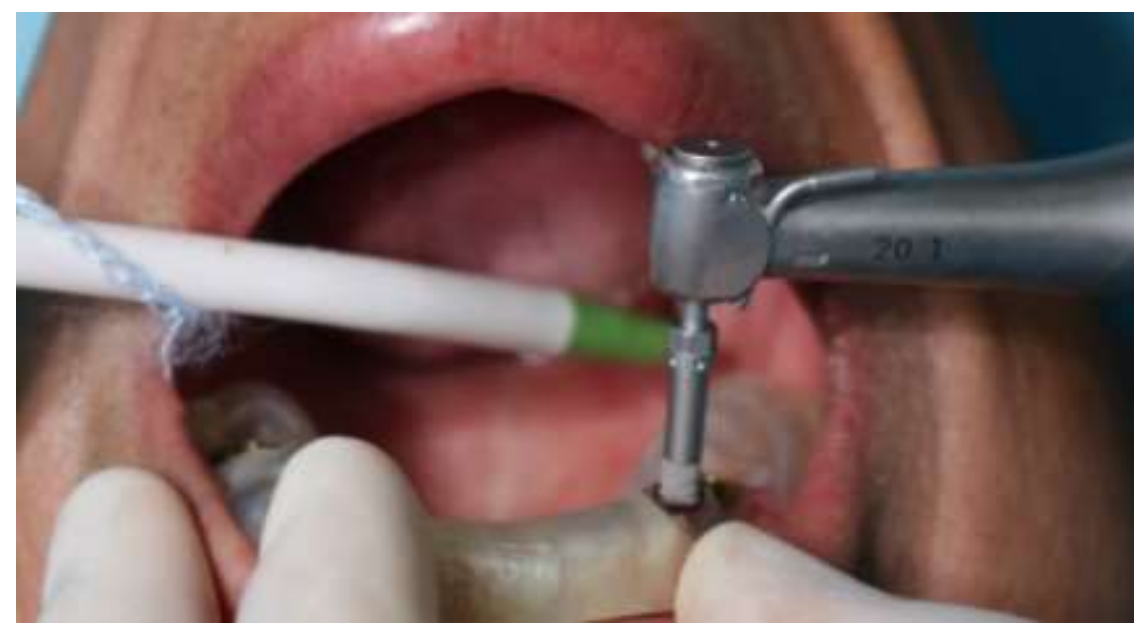

Fonte: Autores (2020). 
Figura 7. Preenchimento do alvéolo com osso particulado mineral bovino (Lumina-Bone Fino 0,5g - Criteria®, São Paulo$\mathrm{SP}$, Brasil) para preenchimento dos espaços intra-alveoar.

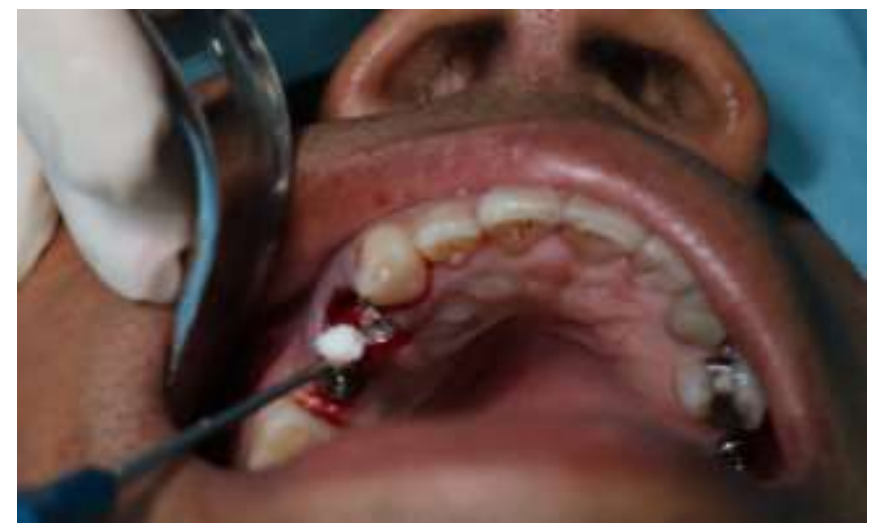

Fonte: Autores (2020).

\section{Carga Imediata Unitária}

Ao obter o travamento na instalação do implante com torque de $45 \mathrm{~N}$, obteve-se a possibilidade de confeccionar uma coroa provisória imediata. Anteriormente a instalação do implante, foi realizado moldagem do arco superior com alginato (Hydrogum 5, Zhermack, Itália), e vazamento do molde em gesso pedra tipo III (Asfer, São Caetano do Sul - SP) obtendo o modelo de estudo superior. Em seguida foi realizado o enceramento e escultura do elemento 14 no modelo em gesso de estudo o qual foi utilizado para escanear e planejar o guia cirúrgico, atendendo os princípios de anatomia dentária. A partir do enceramento prévio, foi confeccionado um molde utilizando silicone de condensação pesada (Clonage, DFL, Rio de JaneiroRJ, Brasil) utilizado para realização do mock-up (Figura 8).

Figura 8. A: Enceramento e escultura do elemento 14 no modelo em gesso de estudo, atendendo os princípios de anatomia dentária. B: Mock-up confeccionado em silicone de condensação pesada para confecção do provisório dentário.



Fonte: Autores (2020).

Sobre o implante foi instalado um munhão universal (3,3x6mm, Altura gengival 3,5mm, Neodent®, Curitiba-PR, Brasil), em seguida posicionado o cilíndro provisório do Munhão Universal, Polímero, 3.3X6 mm (Neodent巴, Curitiba-PR, Brasil) (Figura 9). Posteriomente, a resina bisacrílica (Protemp ${ }^{\mathrm{TM}} 4,3 \mathrm{M}$ ) foi inserida no molde de silicone e o conjunto molde e resina levado em posição intra-oral (Figura 10). Após a polimerização inicial da resina bisacrílica, os excessos foram 
removidos com auxílio de uma sonda exploradora e aguardou-se um total de 5 minutos para que se completasse a polimerização da resina bisacrílica (Figura 11).

Figura 9. Cilíndro provisório de polímero sobre Munhão Universal (3,3x6mm (Neodent®, Curitiba-PR, Brasil) para confecção do provisório dentário.

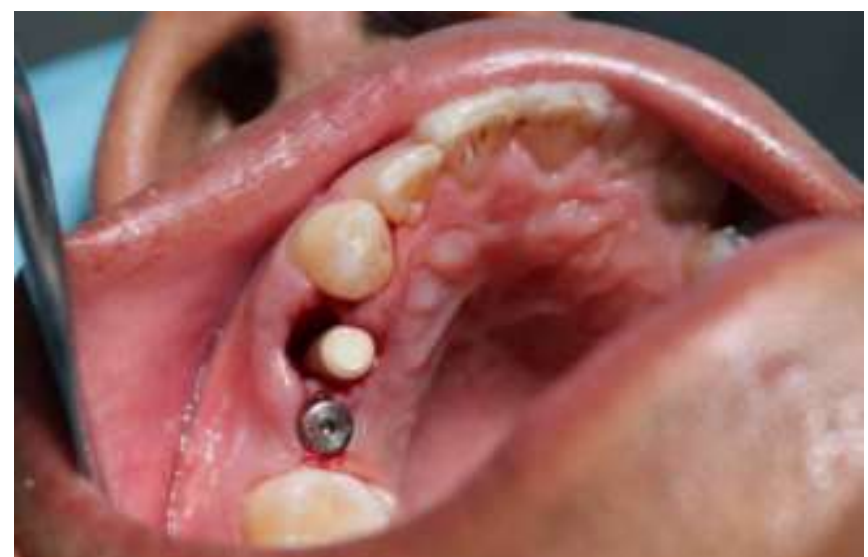

Fonte: Autores (2020).

Figura 10. A: Inserção da resina bisacrílica (Protemp $\left.{ }^{\mathrm{TM}} 4,3 \mathrm{M}\right)$ no molde de silicone; B: conjunto "molde e resina" levado em posição intra-oral sobre cilíndro de polímero para confecção do provisório dentário sobre implante.



Fonte: Autores (2020).

Figura 11. Provisório imediato em resina bisacrílica após polimerização sobre implante intra-oral e visão extra-oral sobre análogo do munhão.



Fonte: Autores (2020). 
No provisório foi adicionado resina composta flow (Opalis Flow, FGM, Santa Catarina, Brasil) nas áreas com bolhas para ajustes estéticos, seguida da remoção dos excessos da resina, e do acabamento e polimento utilizando maxicut e borrachas de polimento em peça reta (American Burrs, Santa Catarina, Brasil) (Figura 12). Em seguida realizada cimentação provisória sem eugenol (Provicol, VOCO, Alemanha). Finalizando com o ajuste oclusal (Figura 13), obtendo-se um resultado funcional e estético (Figura 14).

Figura 12. A: remoção dos excessos da resina bisacrílica no provisório utilizando maxicut; B: realização do acabamento e polimento utilizando borrachas de polimento em peça reta.

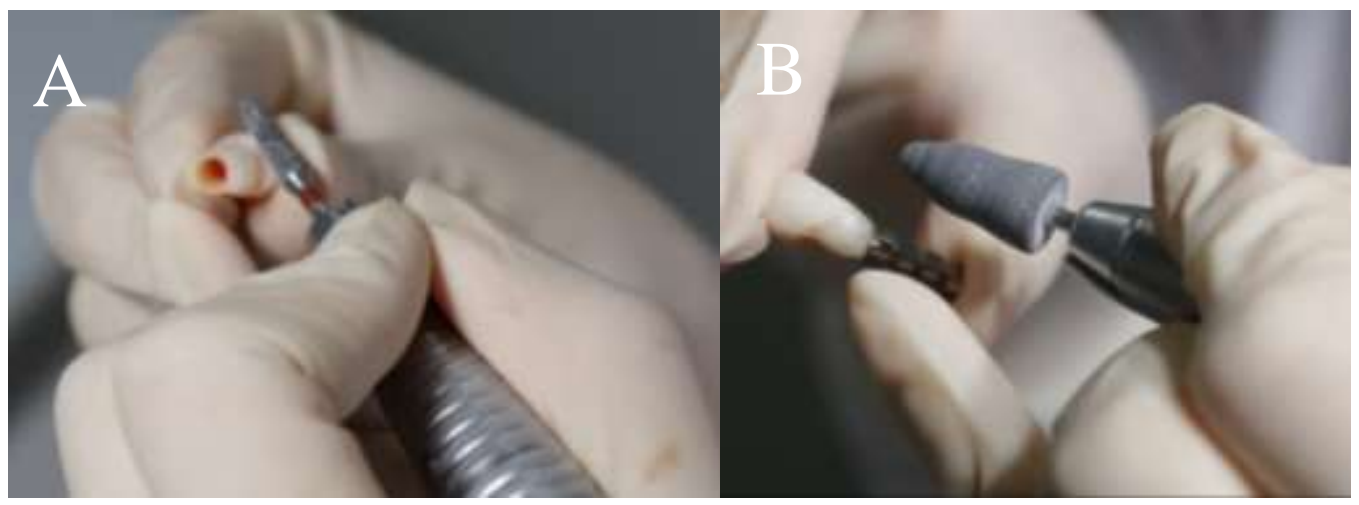

Fonte: Autores (2020).

Figura 13. A: ajuste oclusal do provisório imediato; B: vista oclusal após ajuste.

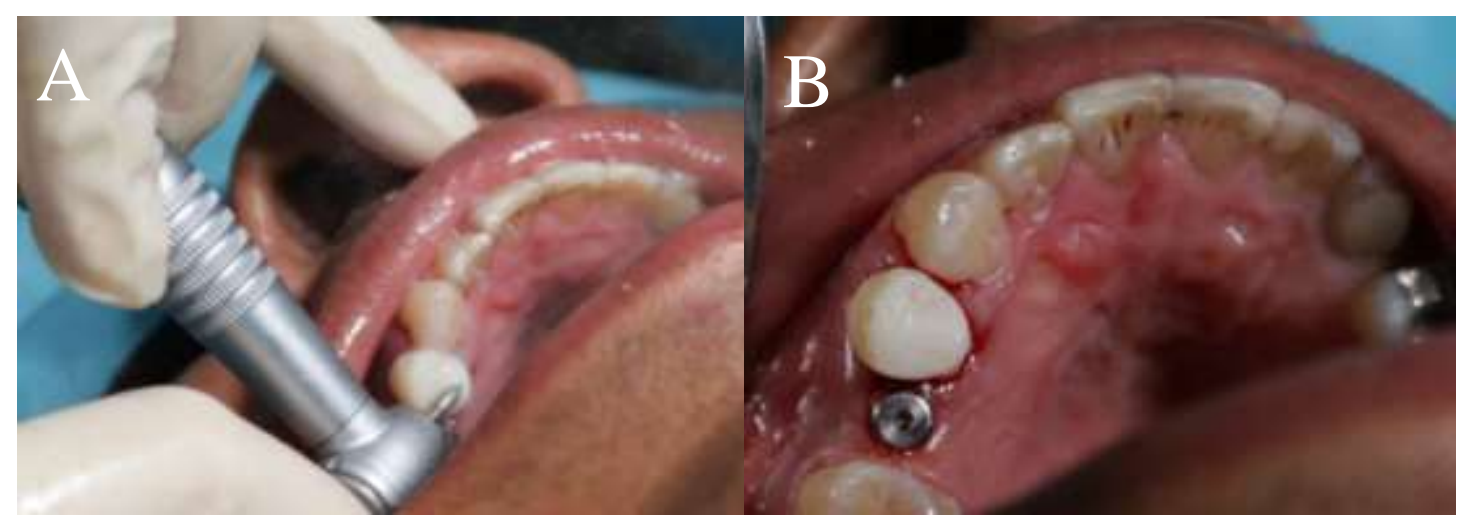

Fonte: Autores (2020).

Figura 14. Coroa sobre implante em área do elemento 14 instalada apresentando resultado funcional e estético.

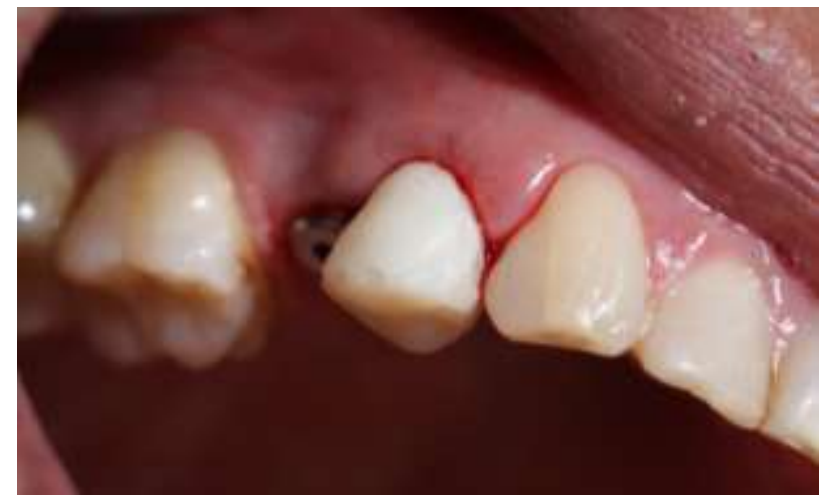

Fonte: Autores (2020). 


\section{Discussão}

O tratamento com implantes tem bom prognóstico, com uma taxa de sucesso em torno de 90\% após 10-15 anos (Schatzle et al., 2004; Holm-Pedersen et al., 2007; Gotfredsen et al., 2008). A instalação de implantes unitários imediatos após a exodontia de dentes apresenta um sucesso previsível (Piattelli, 1993; Popper, H. A., Popper, M. J., \& Popper, J. P., 2003; Oliveira et al., 2010), apresentando vantagens como diminuição do tempo de tratamento e seções cirúrgicas, além de menor reabsorção óssea. (Cauduro et al., 2011). No paciente em questão, a técnica escolhida para tratamento foi implante unitário imediato após exodontia, que consiste em melhor osseointegração pela cicatrização do alvéolo recém extraído.

Entre os demais sistemas de implante, o sistema Cone Morse vem apresentando outras vantagens e alcançando excelentes resultados. Possui algumas características, como a ausência de micro frestas e espaços reduzidos entre o implante e a prótese, alta resistência friccional, plataforma reduzida, diferentes alturas e mínimo deslocamento de pilares, o que permite vantagem nos resultados em implantes imediatos após exodontia (Selistre et al., 2011; Nentwig, 2004; Ribeiro, Araújo, Araújo \& Conti, 2008). No planejamento do caso, o sistema de implante escolhido foi o Cone Morse (Neodent@, Curitiba-PR, Brasil) por apresentar uma série de vantagens entre os demais sistemas.

Para uma reabilitação mais fidedigna, a cirurgia guiada vem com grande avanço tecnológico e trata-se de uma técnica minimamente invasiva. A associação da tomografia computadorizada (TC) a imagens, utilizadas em softwares, possibilita um planejamento virtual tridimensional para instalação de implantes. A técnica apresenta segurança e previsibilidade no tratamento, na maioria dos casos, não possui retalho, onde o guia é posicionado diretamente na mucosa, removendo apenas os tecidos onde o implante será posicionado. Esta técnica permite um planejamento para colocação de implantes em regiões ósseas adequadas, simplificando a fase protética e melhorando o pós-operatório do paciente. (Viana Neto et al., 2009; Strub, Jurdzik \& Tuna, 2012). Na condução prévia do caso, optou-se por planejamento virtual tridimensional com confecção de guia cirúrgico (3D STUDIO - Planejamento Virtual da Face®, Natal-RN, Brasil) para uma estabilidade adequada do implante, permitindo otimizar os resultados estéticos e funcionais.

Implantes guiados submetidos à reabilitação oral por meio de carga imediata se tornou um protocolo com crescentes demandas. (Strub, Jurdzik \& Tuna, 2012). Este tratamento permite a integridade dos tecidos moles adjacentes, preservando osso alveolar e também tecido mole ao redor do implante, além de um excelente resultado estético (Lindeboom et al., 2006). Na reabilitação deste caso clínico, o paciente foi submetido à instalação de implante e carga imediata unitária, simplificando o tratamento reabilitador e devolvendo estética e função, atrelado a um melhor posicionamento do implante pelo uso da cirurgia guiada, podendo ter uma melhor previsibilidade do caso.

\section{Conclusão}

Conclui-se que a reabilitação oral por instalação de implantes através da técnica de cirurgia guiada e da técnica de carga imediata é um procedimento que traz conforto e previsibilidade na reabilitação de perdas dentárias unitárias. A técnica de cirurgia guiada resulta em morbilidade pós-operatória reduzida, permite o planejamento pré-operatório para colocação de implantes em regiões ósseas adequadas, facilitando a reabilitação protética por meio da técnica de carga imediata e devolvendo estética e função para o paciente. A correta indicação, juntamente com um bom planejamento e boa execução de técnica por meio do Cirurgião-Dentista, tem fundamental importância no sucesso do tratamento.

Por fim, sugere-se também que técnica de cirurgia guiada e da técnica de carga imediata anteriormente descrita apresente vantagens, entretanto novo estudos clínicos controlados e randomizados que demonstrem a eficiência e eficácia dessa técnica assim como, seu desempenho clínico em curto, médio e longo prazo. 


\section{Referências}

Borges, A. F., Rego, M. R. D. M., Corrêa, A. M., Torres, M. F., Telles, D. D. M., \& Santiago, L. C. (2014). Planning and treatment in oral rehabilitation with implant-supported prostheses using cephalometric analysis. RGO-Revista Gaúcha de Odontologia, 62(2), $179-184$.

Branemark, P. I., Hansson, B. O., Adell, R., Breine, U., Lindström, J., Hallén, O., \& Ohman, A. (1977). Osseointegrated implants in the treatment of the edentulous jaw. Experience from a 10-year period. Scandinavian journal of plastic and reconstructive surgery. Supplementum, 16, 1-132.

Cauduro, F. S., Silva, D. N., Oliveira, R. B. D., Bridi, M. D. P., Cauduro, D. E., \& Pretto, S. M. (2019). Single tooth morse taper implant placement and restoration immediately after extraction. RGO-Revista Gaúcha de Odontologia, 67.

D’haese, J., Van de Velde, T., Komiyama, A., Hultin, M., \& De Bruyn, H. (2012). Accuracy and complications using computer-designed stereolithographic surgical guides for oral rehabilitation by means of dental implants: a review of the literature. Clinical Implant Dentistry And Related Research, 14(3), 321335 .

Ferreira, J. R. M., Dalapicula, S. S., Conz, M. B., \& Vidigal Junior, G. M. (2007). Enxertos ósseos xenógenos utilizados na implantodontia oral. Implantnews, 303-306.

Gotfredsen, K., Carlsson, G. E., Jokstad, A., Arvidson Fyrberg, K., Berge, M., Bergendal, B., Bergendal, T., Ellingsen, J. E., Gunne, J., Hofgren, M., Holm, B., Isidor, F., Karlsson, S., Klemetti, E., Lang, N. P., Lindh, T., Midtbø, M., Molin, M., Närhi, T., Nilner, K. Danish Society of Oral Implantology (2008). Implants and/or teeth: consensus statements and recommendations. Journal of oral rehabilitation, 35 Suppl 1, 2-8. https://doi.org/10.1111/j.13652842.2007.01833.x

Guerra, M. I. L. (2017). Cirurgia guiada em implantodontia (Doctoral dissertation).

Holm-Pedersen, P., Lang, N. P., \& Müller, F. (2007). What are the longevities of teeth and oral implants?. Clinical oral implants research, 18 Suppl 3, 15-19. https://doi.org/10.1111/j.1600-0501.2007.01434.x

Kayatt, F. E., Kayatt, D. L., \& Junior, I. R. G. (2008). Carga protética imediata ou precoce sobre implante dental osseointegrável: estudo retrospectivo de cinco anos. $R G O, 56(2), 137-142$.

Lindeboom, J. A., Frenken, J. W., Dubois, L., Frank, M., Abbink, I., \& Kroon, F. H. (2006). Immediate loading versus immediate provisionalization of maxillary single-tooth replacements: a prospective randomized study with BioComp implants. Journal of oral and maxillofacial surgery: official journal of the American Association of Oral and Maxillofacial Surgeons, 64(6), 936-942. https://doi.org/10.1016/j.joms.2006.02.015

Muddugangadhar, B. C., Amarnath, G. S., Sonika, R., Chheda, P. S., \& Garg, A. (2015). Meta-analysis of Failure and Survival Rate of Implant-supported Single Crowns, Fixed Partial Denture, and Implant Tooth-supported Prostheses. Journal of international oral health: JIOH, 7(9), $11-17$.

Nentwig G. H. (2004). Ankylos implant system: concept and clinical application. The Journal of oral implantology, 30(3), 171-177. https://doi.org/10.1563/1548-1336(2004)30<171:TAISCA>2.0.CO;2

Oliveira, A., Souza, J., Thomé, G., Melo, A. C., \& Sartori, I. (2010). Implante imediato unitário em função imediata - relato de caso. Revista Da Faculdade De Odontologia - UPF, 13(1). https://doi.org/10.5335/rfo.v13i1.596

Oliveira, M. R., Oliveira, A. D. S., Leite, V. A., Gabrielli, M. A. C., Paganelli, O. E. B., Spin-Neto, R., \& Pereira-filho, V. A. (2017). Immediate loading over lower prosthesis in edentulous mandibles: comparison between two types of prosthetic connection. RGO-Revista Gaúcha de Odontologia, 65(4), 308-314.

Pereira, A. S., et al. (2018). Metodologia da pesquisa científica. [e-book]. Santa Maria. Ed. UAB/NTE/UFSM. https://repositorio.ufsm.br/bitstream/handle/1/15824/Lic_Computacao_Metodologia-Pesquisa-Cientifica.pdf?sequence=1.

Piattelli, A., Ruggeri, A., Franchi, M., Romasco, N., \& Trisi, P. (1993). An histologic and histomorphometric study of bone reactions to unloaded and loaded non-submerged single implants in monkeys: a pilot study. The Journal of oral implantology, 19(4), 314-320.

Popper, H. A., Popper, M. J., \& Popper, J. P. (2003). The Brånemark Novum protocol: description of the treatment procedure and a clinical pilot study of 11 cases. The International journal of periodontics \& restorative dentistry, 23(5), 459-465.

Ribeiro, C. G., Araújo, M. A. R. de, Araújo, C. dos R. P. de, \& Conti, P. C. R. (2008). Provisionalização imediata na região anterior: protocolo clínico para implantes Cone-Morse. ImplantNews, Jan. /fe 2008(1), 13-18.

Rismanchian, M., Bajoghli, F., Gholamreza, T., \& Razavi, M. (2014). Dental implants: early versus standard two-stage loading (animal study). The Journal of oral implantology, 40(1), 84-93. https://doi.org/10.1563/AAID-JOI-D-10-00202

Sanz-Sánchez, I., Sanz-Martín, I., Figuero, E., \& Sanz, M. (2015). Clinical efficacy of immediate implant loading protocols compared to conventional loading depending on the type of the restoration: a systematic review. Clinical oral implants research, 26(8), 964-982. https://doi.org/10.1111/clr.12428.

Schätzle, M., Löe, H., Lang, N. P., Bürgin, W., Ånerud, Å. \& Boysen, H. (2004). The clinical course of chronic periodontitis: IV. Gingival inflammation as a risk factor in tooth mortality. Journal of Clinical Periodontology, 31(12), 1122-1127.

Schnitman, P. A., Wohrle, P. S., \& Rubenstein, J. E. (1990). Immediate fixed interim prostheses supported by two-stage threaded implants: methodology and results. The Journal of oral implantology, 16(2), 96-105.

Selistre, R., de Oliveira, R. B., Cauduro, D. E., Cauduro, M. E., \& Cauduro, F. S. Implantes cone Morse e provisórios imediatos em área estética. Implantodontia, 77, 84

Strub, J. R., Jurdzik, B. A., \& Tuna, T. (2012). Prognosis of immediately loaded implants and their restorations: a systematic literature review. Journal of oral rehabilitation, 39(9), 704-717. https://doi.org/10.1111/j.1365-2842.2012.02315.x 
Research, Society and Development, v. 10, n. 1, e4810110854, 2021

(CC BY 4.0) | ISSN 2525-3409 | DOI: http://dx.doi.org/10.33448/rsd-v10i1.10854

Takahashi, J. M., Dayrell, A. C., Consani, R. L., de Arruda Nóbilo, M. A., Henriques, G. E., \& Mesquita, M. F. (2015). Stress evaluation of implant-abutment connections under different loading conditions: a 3D finite element study. The Journal of oral implantology, 41(2), 133-137. https://doi.org/10.1563/AAIDJOI-D-11-00205

Viana Neto, A., Neves, P. J. C., Madruga, F. A. T., Rocha, R. S., \& Carvalho, R. W. F. D. (2009). Cirurgia guiada virtual para reabilitação oral: revisão de literatura e relato de caso. Rev. cir. traumatol. buco-maxilo-fac, 45-52. 